\title{
Zwischen Erwartungsinflation und Qualitätssicherung im dualen Studium
}

\author{
Ergebnisse einer Kompetenzanalyse für die Ausbildung \\ im kommunalen Finanzwesen
}

Frank Kupferschmidt/Sarah Blum

Die systematische Verzahnung von Theorie und Praxis ist unstrittig der zentrale Erfolgsfaktor dualer Studiengänge, aber gleichzeitig eine ebenso komplexe wie sensible Schnittstelle. Die Autoren erheben und diskutieren am Fallbeispiel des dualen Studiengangs Public Management B.A. Erwartungen aus der Praxis im kommunalen Finanzwesen an dessen Ausbildungsinhalte. In einer im Spannungsfeld zwischen Spezialisierung und Generalisierung konzipierten Befragung bewerteten kommunale Praktiker die Ausbildungsrelevanz von berufspraktischen Kompetenzen. Die Ergebnisse zeigen zum einen eine relativ ausdifferenzierte Bewertung von fachspezifischen Kompetenzen mit einem harten Kern von Fachwissen. Zum anderen verdeutlichen die Ergebnisse die hohe Bedeutung von generalistischen Kompetenzen, die in der Konsequenz eine breite curriculare Entsprechung erfordern. Die Auflösung dieses Zielkonflikts verlangt adäquate Instrumente der Qualitätssicherung für duale Studiengänge, wie sie im aktuellen Forschungsstand vorgeschlagen werden.

\section{Problemaufriss}

Die systematische Verzahnung von Theorie und Praxis ist unstrittig der zentrale Erfolgsfaktor dualer Studiengänge. Die Ausbildung an den beiden Lernorten Hochschule und Praxis gewährleistet einen starken praktischen Anwendungsbezug in den jeweiligen Ausbildungsbetrieben sowie die Vermittlung von

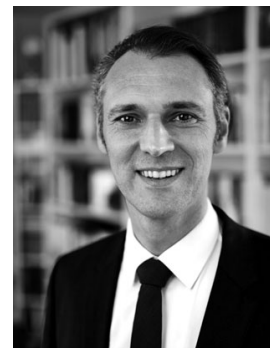

Prof. Dr. Frank Kupferschmidt Professor für Allgemeine Volkswirtschaftslehre und Öffentliche Betriebswirtschaftslehre an der Hochschule für öffentliche Verwaltung und Finanzen Ludwigsburg. betriebsübergreifenden, theoretischen Kompetenzen mit wissenschaftlichem Anspruch. Gleichwohl stellt die Zusammenarbeit von Hochschule und Praxis nicht nur das Erfolgsrezept der dualen Ausbildung dar, sondern ist zugleich eine besonders komplexe und sensible Schnittstelle. ${ }^{1}$

Ein Konfliktpotenzial kann aus den unterschiedlichen Erwartungen der beiden

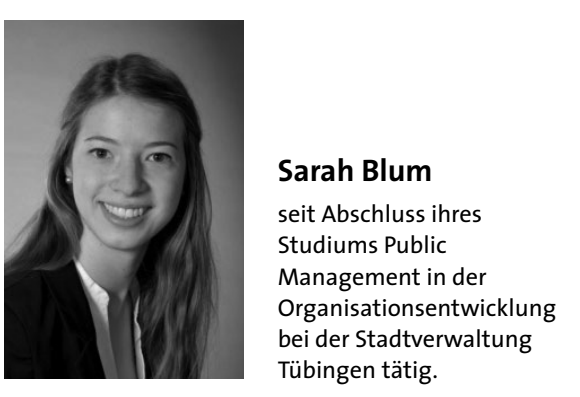

Partner an die Ausbildungsinhalte in dualen Studiengängen entstehen. ${ }^{2}$ Zum einen besteht aus Sicht des Lernorts Hochschule die Gefahr einer Erwartungsinflation, die von den Ausbildungsbetrieben an die Ausbildung herangetragen werden könnte. Dabei werden die Hochschulen mit kurzfristig wechselnden, häufig zusätzlichen und eher betriebsspezifischen Erwartungen an die Ausbildungsinhalte konfrontiert. Aus der Perspektive der Hochschullehrenden können diese das Ausbildungsziel verwässern und mit der Wahrung des wissenschaftlichen Anspruchs kollidieren. Zum anderen besteht aus Sicht des Lernorts Praxis die latente Gefahr einer zu starken Akademisierung, indem sich die Ausbildung an den Hochschulen von der praxisorientierten Lehre entfernt und sich somit die Berufsfähigkeit der Absolventen und Absolventinnen (employability) aus der Perspektive der Praxis verschlechtert.

Im Ergebnis besteht für die duale Ausbildung ein wesentlicher Erfolgsfaktor darin, den schwierigen Spagat zwischen der Wahrung des wissenschaftlichen Anspruchs und der Gewährleistung der unmittelbaren Berufsfähigkeit der Absolventinnen und Absolventen zu bewältigen. Dieser Beitrag greift die einführend beschriebene Problemstellung am Beispiel des Studiengangs Public Management B.A. in Baden-Württemberg an der Hoch-

Hesser/Langfeldt 2017, S. 71.

2 Vgl. Ausführungen von Gerhards 2019 in dieser Zeitschrift zu den "Schattenseiten des dualen Studiums“ am Beispiel der Dualen Hochschule Baden-Württemberg DHBW. 
schule für öffentliche Verwaltung und Finanzen Ludwigsburg auf. ${ }^{3}$ Die Autoren erheben und analysieren die Erwartungen von Praktikern an die Ausbildungsinhalte im kommunalen Finanzwesen, die für eine Tätigkeit in Kämmereien bzw. für die Aufgaben eines Fachbediensteten für das Finanzwesen auf Grundlage von $\$ 116$ der baden-württembergischen Gemeindeordnung qualifizieren sollen. Mit den Ergebnissen aus der zugrundeliegenden Befragung wird die Diskussion um Qualitätssicherung der Ausbildung im kommunalen Finanzwesen auf eine aktuelle und objektive Basis gestellt. Die Erkenntnisse nanzbereichs besteht. Die interne Validität der Erhebung ist insofern gewährleistet, als mit dem zielgerichteten Versand des Fragebogens an Beschäftigte aus dem kommunalen Finanzbereich in den Mitgliedskommunen des Städtetags BadenWürttemberg vergleichbare Befragungsteilnehmer ausgewählt wurden. ${ }^{5}$ Der Rücklauf lag bei 82 Prozent, die Grundgesamtheit der Befragung umfasst 93 Personen. Überwiegend ist die Position des Amts- und Fachbereichsleiter mit 81 Prozent in der Stichprobe vertreten. Nahezu zwei Drittel der Befragten sind seit mehr als sechs Jahren im Amt und rund 85 Pro-

\section{"Erfolgsfaktor für die duale Ausbildung ist es, den schwierigen Spagat zwischen Wissenschaftlichkeit und Berufsfähigkeit zu bewältigen."}

der Befragung werden anschließend im Rahmen der Problemstellung diskutiert und in den aktuellen Forschungsstand zur Qualitätssicherung in der dualen Ausbildung eingebettet.

\section{Methodik der Untersuchung}

Aktualität und Relevanz dieser Untersuchung bestehen darin, dass zum einen die Kämmereien besonders stark von Nachwuchsmangel betroffen zu sein scheinen und zum anderen mit diesem Studienabschluss die erforderliche gemeinderechtliche Qualifikation für das Führen eines kommunalen Haushalts erworben wird. ${ }^{4}$ Um die Vielfalt der baden-württembergischen Kämmereien in den 44 Stadt- und Landkreisen sowie den 1.100 Kommunen verschiedenster Größenklassen in der Datenerhebung zu erfassen, erschien zum einen eine möglichst große und weit gestreute Stichprobe als vorteilhaft. Zum anderen führt die Fragestellung zu einer relativ klar definierten Stichprobe, die aus Beschäftigten des kommunalen Fi- zent sind selbst Absolventen bzw. Absolventinnen des Studiengangs. ${ }^{6}$

Die Erhebung sollte eine möglichst hohe Vergleichbarkeit der Daten und Merkmalsausprägungen erreichen, so dass sich der Fragebogen als geeignete Untersuchungsmethode darstellte. Die Befragung mit einem online-basierten Fragebogen mit insgesamt 84 Items wies mit 86

3 Der Studiengang Public Management B.A. ist ein praxisintegrierter Studiengang, bei dem die Studierenden über die gesamten Ausbildungszeit vier Semester an der Hochschule studieren. Die praktische Ausbildung erfolgt in einem vorgeschalteten, halbjährigen Einführungspraktikums sowie während einer insgesamt 15-monatigen praktischen Ausbildungsphase, die die Studierenden in vier unterschiedlichen Ausbildungsstellen absolvieren.

4 Die kommunale Haushaltswirtschaft ist als solches nur an den Hochschulen für den öffentlichen Dienst Studieninhalt. Das Fehlen eines fachlich korrespondierenden Universitätsstudiums ist eine wesentliche, strukturelle fachliche Eigenart des kommunalen Finanzmanagements. Weder die Rechts- noch die Wirtschaftswissenschaften befassen sich im Kern mit dem kommunalen
Prozent geschlossenen Fragen einen sehr hohen Standardisierungsgrad auf und gewährleistet die Objektivität der Erhebung. In der Konstruktion des Fragebogens wurden jene Kompetenzen zusammengetragen, die das Berufsfeld im kommunalen Finanzwesen widerspiegeln. Dafür wurden Modulhandbücher verschiedenster Hochschulen für öffentliche Verwaltung in Deutschland sowie einschlägige Stellenanzeigen von Kommunen verwendet.

Schwerpunkt der Befragung waren mit 37 Items die spezifischen Fachkenntnisse (finanzwirtschaftliche Kompetenzen), die mit 66 Prozent zwei Drittel des geschlossenen Frageteils ausmachen. Die weiteren 19 bzw. 34 Prozent der Items umfassen übergreifende bzw. generalistische Kompetenzen, die in vier Kategorien Übergreifendes Wissen, Methoden-, Personal- und Führungskompetenzen sowie technische Kompetenzen gegliedert wurden.

Für die geschlossenen Fragen wurde durchgängig eine fünfstufige, bipolare Ratingskala mit Mittelkategorie verwendet. Dadurch können die Bewertungen übergreifend über die Kompetenzbereiche verglichen werden. Aus der fünfstufigen Skala der geschlossenen Fragen wurde eine Klassierung der Mittelwerte in drei Relevanzklassen vorgenommen. Als „sehr relevant" gelten Kompetenzen mit einem Mittelwert größer 4,0, als „relevant“ zwischen 3,0 und 4,0 und als „weniger relevant" mit einem Mittelwert kleiner 3,0. Die Varianz der Ergebnisse wurde über die Standardabweichung berücksichtigt.

Wirtschaftsrecht bzw. der Finanzwirtschaft in Kommunen oder Eigenbetrieben. Aufgaben sind insbesondere Haushaltsplanung, Haushaltsüberwachung und die Jahresrechnung.

5 Die Befragung wurde an die Zielgruppe, die AGKämmerer/-innen mit insgesamt 114 Mitgliedern des Städtetags Baden-Württemberg, versandt.

6 Der Rücklauf spiegelt die hohe Relevanz der Fragestellung bei den Ausbildungsstellen wider. Das Interesse am arbeits- und zeitintensiven Austausch zwischen Hochschule und Praxis kann nicht zwingend vorausgesetzt werden: in einer Studie von Langfeldt geben nur rund 40 Prozent der an dualen Studiengängen beteiligten Unternehmen an, ein starkes Interesse an der Mitgestaltung des Curriculums bzw. der Praxismodule zu haben, Langfeldt 2018, S. 8. 


\section{Erwartungen an spezifische Fachkompetenzen}

Die finanzwirtschaftlichen Kompetenzen sind für die curriculare Verzahnung wesentlich und stehen daher im Fokus der Befragung. Die 37 ausgewählten Items beschreiben detailliert spezifische Themengebiete und Aufgabenstellungen, die für das kommunale Finanzwesen maßgeblich sind. Abbildung 1 zeigt die Ergebnisse der Befragung, die anhand der Mittelwerte in die drei Relevanzklassen gegliedert und innerhalb der Gruppen ordinal dargestellt sind. Der grafische Überblick verdeutlicht, dass die Befragten im Ergebnis eine ausdifferenzierte Bewertung der Fachkompetenzen über die Relevanzklassen vorgenommen haben.

Zehn von 37 Kompetenzen werden als weniger relevant eingeordnet. Dazu zählen eher spezifische Kenntnisse wie Liegenschaftscontrolling, etwas überraschend die Anwendung des Vergaberechts, aber auch Kenntnisse der EPSAS oder die Themen Wirtschaftsförderung, Standortmarketing oder das Erstellen von Marketingkonzepten.

In die mittlere Relevanz wird rund die Hälfte der Kompetenzen eingeordnet. Dazu zählen „Kenntnisse im Bereich öffentlicher Unternehmen und Beteiligungen“, der „Rechtsformen kommunaler Aufgabenerfüllung" sowie das thematisch dazugehörige „Wissen über die verschiedenen Formen der interkommunalen Zusammenarbeit“. Im Hinblick auf die Vielzahl und Verschiedenheit der Ausgliederung kommunaler Aufgaben in privatwirtschaftlichen Unternehmen und interkommunalen Verbänden erscheint die Einordnung dieser Items durch die Praktiker nachvollziehbar. Etwas überraschend werden die Kompetenzen „Satzungsrecht“, „Beitragsrecht und -kalkulation“, und „Gesamtabschluss“ eher als moderat relevant innerhalb dieser Gruppe eingeordnet.

Unter den sehr relevanten Kompetenzen erscheinen zehn von 37 Kompetenzen, die zum klassischen Brot-und-ButterGeschäft in Kämmereien zählen dürften. „Aufstellung, Vollzug und Überwachung der Haushaltspläne nach NKHR“, „Analyse der Ertrags-, Vermögens- und Fi-

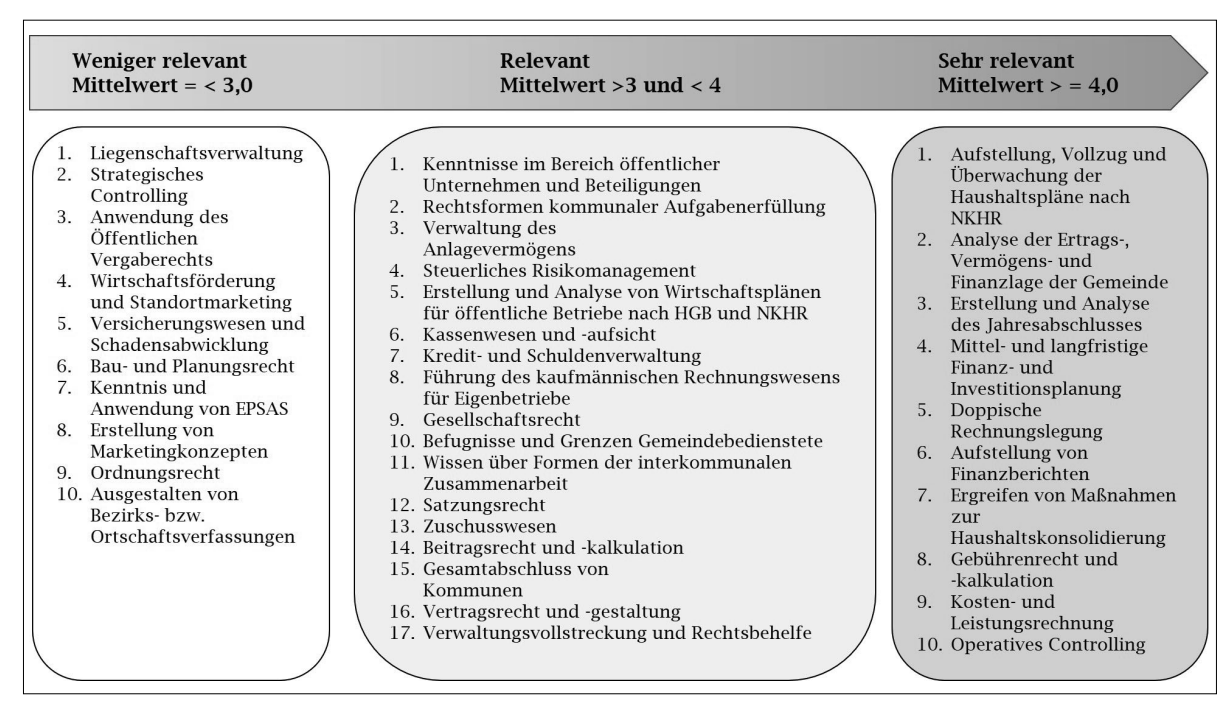

Abb. 1: Einordnung der finanzwirtschaftlichen Kompetenzen

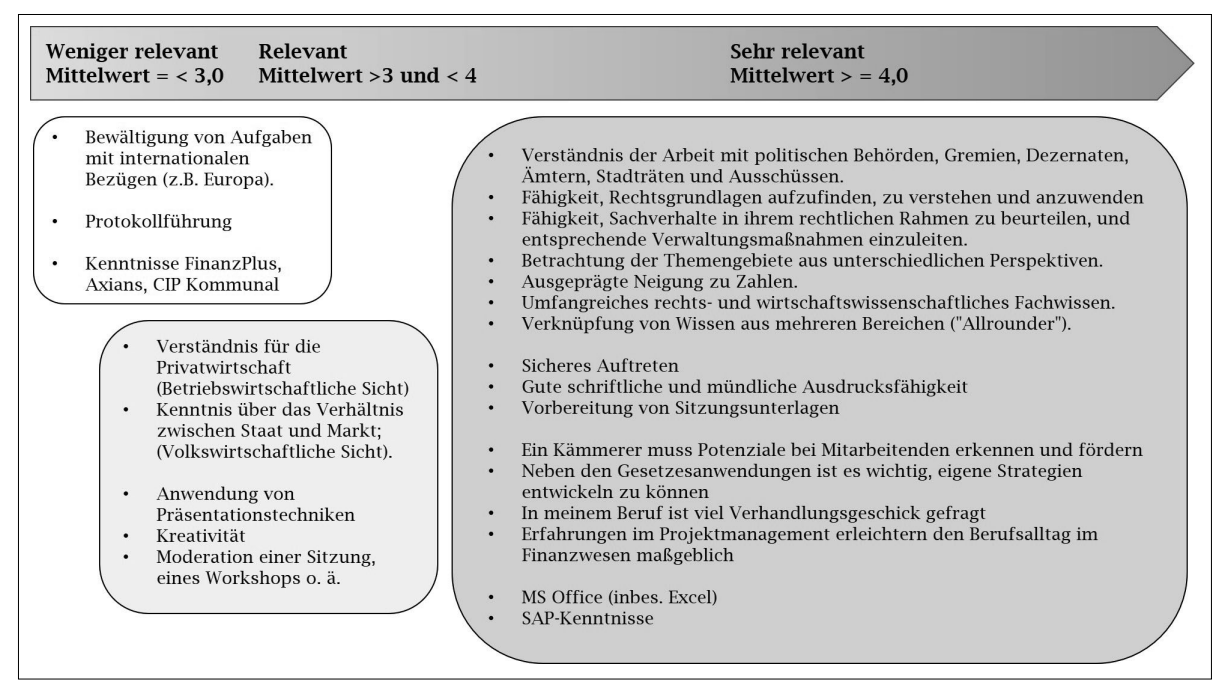

Abb. 2: Elnordnung der fachübergreifenden Kompetenzen

nanzlage der Gemeinde“ sowie die beiden Items „Erstellung und Analyse des Jahresabschlusses“ und „Mittel- und langfristige Finanz- und Investitionsplanung “ betreffen Kerngebiete des kommunalen Haushaltsrechts.

Insgesamt erscheinen die gewonnenen Ergebnisse insofern plausibel, als die am höchsten bewerteten Kompetenzen sich weitestgehend mit den klassischen Aufgabenbereichen in Kämmereien decken. Unter den weniger relevant eingestuften Items finden sich eher spezifische Kompetenzen, die weniger häufig unter das klassische Alltagsgeschäft fallen dürften.

\section{Erwartungen an fachübergreifen- de Kompetenzen}

In der weiteren Befragung wurde die Relevanz von insgesamt 19 fachübergreifenden bzw. generalistischen Kompetenzen abgefragt. Abbildung 2 zeigt die Ergebnisse überblickend in der bereits bei Abbildung 1 beschriebenen Systematik. Aus der Grafik wird deutlich, dass die Praktiker die übergreifenden Kompetenzen weniger ausdifferenziert, sondern im Unterschied zu den fachspezifischen Kompetenzen relativ häufig als sehr relevant einordnen.

Die 19 fachübergreifenden Kompetenzen wurden in der Befragung nach übergreifendem Wissen, Methoden-, Führungsund Sozialkompetenzen und technischen Kompetenzen differenziert. Im Kompetenzbereich des „Übergreifenden Wissens“ wurden zehn Fähigkeiten und Fertigkeiten abgefragt, die mehrere Themenfelder verknüpfen, wie beispielsweise die am höchsten bewertete Kompetenz „Verständnis der Arbeit mit politischen Behörden, Gremien, Dezernaten, Ämtern, Stadträten und Ausschüssen“. Auffällig ist, dass die Praktiker in dieser Untergruppe sieben 
Kupferschmidt/Blum, Zwischen Erwartungsinflation und Qualitätssicherung im dualen Studium

\begin{tabular}{|c|l|c|c|}
\hline Nr. & Kompetenz & Mittelwert & Kompetenzbereich \\
\hline T1 & Microsoft Excel & 4,8 & Technische Kompetenz \\
\hline T2 & $\begin{array}{l}\text { Aufstellung, Vollzug und Überwachung der Haushaltspläne } \\
\text { nach NKHR }\end{array}$ & 4,8 & Finanzwirtschaftliche Kompetenz \\
\hline T3 & Sicheres Auftreten & 4,7 & Methodenkompetenz \\
\hline T4 & Gute schriftliche und mündliche Ausdrucksfähigkeit & 4,7 & Methodenkompetenz \\
\hline T5 & $\begin{array}{l}\text { Analyse der Ertrags-, Vermögens- und Finanzlage der } \\
\text { Gemeinde }\end{array}$ & 4,7 & Finanzwirtschaftliche Kompetenz \\
\hline T6 & $\begin{array}{l}\text { Ein Kämmerer muss Potenziale bei Mitarbeitenden erkennen } \\
\text { und fördern. }\end{array}$ & 4,7 & Führungs- und Sozialkompetenzen \\
\hline T7 & $\begin{array}{l}\text { Neben der Gesetzesanwendung ist es wichtig, eigene } \\
\text { Strategien entwickeln zu können. }\end{array}$ & 4,6 & Führungs- und Sozialkompetenzen \\
\hline T8 & Erstellung und Analyse des Jahresabschlusses & 4,6 & Finanzwirtschaftliche Kompetenz \\
\hline T9 & Mittel- \& langfristige Finanz- und Investitionsplanung & 4,6 & Finanzwirtschaftliche Kompetenz \\
\hline T10 & $\begin{array}{l}\text { Verständnis der Arbeit mit politischen Behörden, Gremien, } \\
\text { Dezernaten, Ämtern, Stadträten und Ausschüssen. }\end{array}$ & 4,5 & Übergreifende Kompetenz \\
\hline
\end{tabular}

Tab. 1: Top 10 der Kompetenzen über alle Kompetenzbereiche

\begin{tabular}{|l|l|c|c|}
\hline Nr. & Kompetenz & Mittelwert & \multicolumn{1}{|c|}{ Kompetenzbereich } \\
\hline L10 & Ordnungsrecht (z.B. Polizeirecht) & 1,6 & Finanzwirtschaftliche Kompetenz \\
\hline L9 & Ausgestalten von Bezirks- bzw. Ortschaftsverfassungen & 1,6 & Finanzwirtschaftliche Kompetenz \\
\hline L8 & Erstellung von Marketingkonzepten z.B. Stadtmarketing & 1,8 & Finanzwirtschaftliche Kompetenz \\
\hline L7 & $\begin{array}{l}\text { Kenntnis und Anwendung der Europäischen Rechnungs- } \\
\text { legungsstandards für den öffentlichen Sektor (EPSAS) }\end{array}$ & 2,0 & Finanzwirtschaftliche Kompetenz \\
\hline L6 & Bau- und Planungsrecht & 2,0 & Finanzwirtschaftliche Kompetenz \\
\hline L5 & Protokollführung & 2,1 & Methodenkompetenz \\
\hline L4 & Versicherungswesen und Schadensabwicklung & 2,4 & Finanzwirtschaftliche Kompetenz \\
\hline L3 & Wirtschaftsförderung und Standortmarketing & 2,4 & Finanzwirtschaftliche Kompetenz \\
\hline L2 & Aufgaben mit internationalen Bezügen (z.B. Europa) & 2,5 & Übergreifende Kompetenz \\
\hline L1 & $\begin{array}{l}\text { Anwendung des Öffentlichen Vergaberechts (z.B. Erstellung } \\
\text { von Leistungsbeschreibungen, Umgang mit Wertungskrite- } \\
\text { rien) }\end{array}$ & 2,6 & Finanzwirtschaftliche Kompetenz \\
\hline
\end{tabular}

Tab. 2: Low 10 der Kompetenzen über alle Kompetenzbereiche

von zehn Kompetenzen als sehr relevant einstufen. Lediglich die Kompetenz „Bewältigung von internationalen Aufgaben, z.B. Europa" wurde als weniger relevant eingeordnet.

Unter Methodenkompetenzen wurden sieben Tätigkeiten und persönliche Merkmale abgefragt, die insbesondere einschlägigen Stellenausschreibungen entnommen wurden. Hier wurden nur die drei Kompetenzen „Sicheres Auftreten“, "Gute schriftliche und mündliche Ausdrucksfähigkeit“ sowie das „Vorbereiten von Sitzungsunterlagen“ als sehr relevant eingeordnet.

Unter „Führungs- und Sozialkompetenzen" wurden vier weiche Faktoren des Berufsfeldes aufgeführt. Alle Kompetenzen wurden durchgängig als „sehr relevant“ bewertet: „Ein Kämmerer muss Potenziale bei Mitarbeitenden erkennen und fördern“, „Neben der Gesetzesanwendung ist es wichtig, eigene Strategien entwickeln zu können“, „Verhandlungsgeschick“ und „Erfahrungen im Projektmanagement". Diese Ergebnisse sind zu relativieren, da eine gewisse Vorabselektion der Fragen aufgrund der Stellenausschreibungen erfolgte.

Schließlich wurde unter „Technische Kompetenzen“ die Relevanz von acht Anwendungen abgefragt. Die Befragten ordneten die Anwendungen Excel und der weiteren MS-Office-Anwendungen Outlook, Word und Power Point sowie SAPKenntnisse als sehr relevant ein.

\section{Verdichtung der Ergebnisse auf Top-10 und Low-10-Komptenzen}

Für eine Verdichtung der Ergebnisse werden zunächst die zehn relevantesten Kompetenzen aus allen Kategorien anhand des jeweiligen Bewertungswerts in Tabelle 1 zusammengefasst. ${ }^{7}$ Unter den Top-10-Kompetenzen haben die befragten Praktiker vier finanzwirtschaftliche
Kompetenzen, zwei Methodenkompetenzen, zwei Führungsund Sozialkompetenzen sowie jeweils eine technische und übergreifende Kompetenz eingeordnet. Die Top 10 erscheinen relativ heterogen aus den Kompetenzbereichen zusammengesetzt $\mathrm{zu}$ sein, allerdings fällt der überproportionale Anteil von übergreifenden gegenüber den fachspezifischen Kompetenzen auf.

Tabelle 2 zeigt die zehn am wenigsten relevanten Kompetenzen über alle Felder aus Sicht der Praktiker. Unter diese Low-10-Kompetenzen haben die befragten Praktiker acht finanzwirtschaftliche Kompetenzen sowie jeweils eine Methoden- und übergreifende Kompetenz eingeordnet. Damit sind die fachspezifischen Kompetenzen in den Low 10 überproportional vertreten.

\section{Management der Erwartungs- inflation: Spezialisierung oder Generalisierung?}

Die oben dargestellten Einzelergebnisse lassen sich im Kontext der eingangs formulierten Fragestellung diskutieren, inwieweit diese auf das typische Spannungsfeld der Erwartungsinflation an duale Studiengänge aus den beiden Lernorten Hochschule und Praxis hindeuten.

7 Zusätzlich wurden die Relevanzwerte nach den Größenklassen der Kommunen differenziert. Jedoch zeigt diese Differenzierung lediglich geringe Abweichungen vom Gesamtwert. Bei den Ergebnissen der Verwaltungen mit $\leq 15.000 \mathrm{EW}$ ergeben sich zwar anteilig die meisten Differenzen zum Gesamtmittelwert, allerdings nur in der Schwankungsbreite von $\pm 0,1$ Punkten und in drei Fällen von $\pm 0,2$ Punkten. 
Prima facie sprechen sich die befragten Praktiker im offenen Frageteil wenig überraschend für eine Stärkung der praxisbezogenen Ausrichtung der Ausbildung aus. Gemessen an den Ergebnissen des geschlossenen Frageteils deuten sich zwei Muster aus: zunächst erscheint das Grundlagenwissen zur Struktur und den Aufgaben des kommunalen Finanzwesens von wesentlicher Bedeutung. Die sieben finanzwirtschaftlichen Kompetenzen mit der höchsten Relevanzbewertung (s. Tab. 1) sind unmittelbar dem Neuen Kommunalen Haushaltsrecht (NKHR) zuzuordnen, was wiederum dem typischen Arbeitsumfeld in Kämmereien entspricht. Hingegen nimmt die durchschnittliche Re- schen Ansatzes des Studiengangs ist insofern bemerkenswert, als sich die Stichprobe prima facie einerseits aus Spezialisten zusammensetzt und andererseits für das Aufgabenfeld im kommunalen Finanzwesen hohes Expertenwissen vorausgesetzt wird.

\section{Einbettung in die Qualitätssicherung}

Die Erhebung liefert nicht nur studiengangsspezifische Erkenntnisse, die in den befassten Expertenkreisen und Gremien diskutiert werden können. Vielmehr können die Ergebnisse auch im Rahmen der vorhandenen Literatur zur Qualitätssi-

\section{"Sowohl Generalisierung als auch Spezialisierung in einer definierten, nicht vermehrbaren Studienzeit zu erreichen, erscheint als klassischer Zielkonflikt."}

levanz bei stärker spezifischen Fachkompetenzen $\mathrm{ab}$ und führt insgesamt zu dem sehr ausdifferenzierten Kompetenztableau aus Abbildung $1 .^{8}$

Gleichzeitig werden übergreifende bzw. generalistische Kompetenzen von den Befragten überproportional als sehr relevant eingeordnet. Die hohe Relevanz von rund der Hälfte der fachübergreifenden Kompetenzen (s. Abb. 2) sowie der überproportionale Anteil von nicht fachspezifischen Kompetenzen in den Top 10 unterstützt die Bedeutung generalistischer Kompetenzen. Diese veranschaulicht sich auch in der ausnahmslos hohen Relevanz von Führungskompetenzen, von denen zwei zudem in den Top-10-Kompetenzen genannt wurden. Auch in einer offenen Frage zu weiteren Anregungen wird die generalistische Ausrichtung des Studiums in 14 relevanten Anmerkungen (entspricht $15 \%$ der Befragten) bekräftigt. Die relativ starke Unterstützung des generalisti- cherung in der dualen Ausbildung eingeordnet werden. Vielfältige und zum Teil empirisch gestützte Empfehlungen bieten insbesondere die Studie von Hesser/ Langfeldt, das Handbuch „Qualitätsentwicklung im dualen Studium“ sowie das Positionspapier des Wissenschaftsrats. ${ }^{9}$ Zudem hat der Akkreditierungsrat Empfehlungen für duale Studiengänge erarbeitet, um deren Besonderheiten in Akkreditierungsverfahren entsprechend $\mathrm{zu}$ berücksichtigen. ${ }^{10}$

Eine wesentliche Qualitätssicherungsmaßnahme ist die standardisierte Evaluation der Ausbildung, die nicht nur klassische Lehrveranstaltungen an den Hochschulen, sondern auch praktische Ausbildungsabschnitte umfassen sollte. Konkret sollte diese Erkenntnisse zur inhaltlichen Verzahnung selbst, zur Lernortkooperation aus Sicht der Studierenden sowie zum Fortschritt des Kompetenzerwerbs der Studierenden liefern können.
Dabei ist die Konzeption einer geeigneten Methode - wie auch die oben beschriebene Befragung erwies - angesichts der Heterogenität von Aufgaben und Ausbildungsstellen nicht trivial und erfordert nennenswerte Ressourcen.

Ein weiteres wesentliches Element der Verzahnung ist die Reflexion der praktischen Ausbildung durch den Studierenden und deren konkreten Umsetzung in ein geeignetes didaktisches Format. Dies umfasst ex ante die verbindliche und umfassende Definition von Lernzielen der praktischen Ausbildung in den Modulhandbüchern, die das inhaltliche Niveau der Praxisphasen beschreiben, jedoch auch der Heterogenität der Ausbildungsstellen Rechnung tragen müssen. Zudem denkbar wären Kataloge für die Eignung von Kooperationspartnern sowie in Analogie zu Betreuungsverträgen in der Privatwirtschaft standardisierte Dokumente zu den Rechten und Pflichten der Kooperationspartner. Sowohl Hesser/Langfeldt als auch Meyer-Guckel et al. empfehlen ex post verbindliche Ausbildungsdokumentationen. ${ }^{11}$

Die Qualitätssicherung umfasst selbstredend auch die Lehr- und Lernprozesse an der Hochschule. In der Hochschullehre wäre die Förderung didaktischer Kompetenzen zur Einbindung praktischer Erfahrungen bei hauptamtlichen Lehrpersonen ein denkbarer Ansatz. Meyer-Guckel et al. beschreiben mit dem an der FH Bielefeld entwickelten ESRIA-Modell ein mögliches Methodenkompendium, das Anregungen für die Einbindung von in der Berufspraxis erworbenem Wissen der Studierenden in die Hochschullehre bietet. Darüber hinaus wäre es denkbar, konkrete Lehrveran-

8 Die Experten scheinen sich zudem einig zu sein, da die Bewertungen eine relativ geringe Standardabweichung zwischen 0,5 und 0,7 aufweisen. Spezifische Kompetenzen außerhalb dieser klassischen Kernkompetenzen werden hingegen eher schwächer bewertet. Dies verdeutlich auch der relativ hohe Anteil von spezifischen Kompetenzen unter den Low 10, wie beispielsweise Ordnungsrecht, Bau- und Planungsrecht, Vergaberecht oder Stadtmarketing bzw. Marketingkonzeption.

9 Hesser/Langfeldt 2017; Meyer-Guckel et al. 2015 und Wissenschaftsrat 2013.

10 Akkreditierungsrat 2010.

11 Hesser/Langfeldt 2017, 72f. und Meyer-Guckel et al. 2015, S. 84 . 
staltungsformate zur Praxisreflexion zu entwickeln. ${ }^{12}$

Kuhlee/Irmscher sehen die Lernortkooperation in der dualen Ausbildung als ein wesentliches Problemfeld: „Häufig unzureichend strukturierte und institutionalisierte Zusammenarbeit zwischen den Lernorten scheint eine Vielzahl dualer Studienangebote zu prägen “. ${ }^{13}$ Der Wissenschaftsrat empfiehlt nachdrücklich die Einrichtung gemeinsamer Gremien zum inhaltlichen, unmittelbaren und regelmäßigen Austausch. Meyer-Guckel et al. beschreiben Erfahrungen an der HAW München mit der Einrichtung von Qualitätszirkeln als qualitätssichernde Maß- leistung des wissenschaftlichen Anspruchs und der Gewährleistung der employability der Studierenden in dualen Studiengängen. Die Umsetzung der eher ambivalenten Ergebnisse in ein Curriculum unter der Voraussetzung ist nicht trivial: sowohl Generalisierung als auch Spezialisierung in einer definierten, nicht vermehrbaren Studienzeit $\mathrm{zu}$ erreichen, erscheint als klassischer Zielkonflikt.

Denn einerseits bedeutet die Anforderung, die fachlichen Kernkompetenzen im kommunalen Finanzmanagement in der Ausbildung zu stärken, eben konkret einen entsprechenden curricularen Umfang. Andererseits untermauert die Forderung ben bzw. Ausbildungsstellen erscheinen. Jedoch erfordert dieses Lernziel einen ebenso adäquaten Anteil am Curriculum des Studiengangs.

Für die Umsetzung erscheint es zunächst zentral, dass beide Lernorte ein gemeinsames Verständnis für den grundlegenden Markenkern des Studiengangs entwickeln. Dieser kann als Maßstab für die Bewertung dienen, mit welchen Kompetenzen der wissenschaftliche Anspruch des Studiums gewährleistet werden kann, der wiederum nach Auffassung des Wissenschaftsrats an erster Stelle stehen sollte ${ }^{16}$. Sonst lässt sich der schwierige Spagat zwischen Generalisierung und Spezialisierung bzw. zwischen wissenschaftlichen Anspruch und Praxisbezug nicht meistern.

\section{Literatur}

Akkreditierungsrat (2010): Handreichung der AG „Studiengänge mit besonderem Profilanspruch".

Gerhards, R. (2019): Schattenseiten des dualen Studiums?, in: Verwaltung \& Management 25. Jg., 6, S. 292-296.

Hesser, W./Langfeldt, B. (2017): Das duale Studium aus Sicht der Studierenden, Hamburg.

Krone, S. (2015): Neue Karrierepfade in den Betrieben: Nachwuchsbindung oder Akademisierung? In: Krone, S. (Hrsg.): Dual Studieren im Blick. Entstehensbedingungen, Interessenlagen und Umsetzungsverfahren in dualen Studiengängen. Wiesbaden, 51-88.

Kuhlee, D./Irmscher, M. (2018): Duales Studium vs. duale Ausbildung. Zur Diskussion um die Relevanz dualer Studienangebote unter Berücksichtigung der Unternehmensperspektive, in: bwp Berufs- und Wirtschaftspädagogik Nr. 34/2018, S. 1-24.

Langfeldt, B. (2018): Lernortkooperation im dualen Studium - zu viel oder zu wenig Einfluss der Hochschulen auf die betrieblichen Praxisphasen, in: bwp Berufs- und Wirtschaftspädagogik $\mathrm{Nr}$.34/2018, S. 1-20.

Meyer-Guckel, V., et al. (2015): Qualitätsentwicklung im dualen Studium. Ein Handbuch für die Praxis.

Wissenschaftsrat (2013): Empfehlungen zur Entwicklung des dualen Studiums. Positionspapier.

Praxis umzusetzen ist als die Vermittlung generalistischer Inhalte. Für die Vermittlung der übergreifenden und generalistischen Kompetenzen in der Breite des Fächerkanons erscheint prima facie der für die Studierenden einheitliche Lernort Hochschule stärker in der Verantwortung zu stehen, da diese eher weniger geeignet für die praktische Ausbildungsabschnitte in den eher heterogenen einzelnen Betrie-

\footnotetext{
12 Meyer-Guckel et al. 2015, 99 f.

13 Kuhlee/Irmscher 2018, S. 11.

14 Wissenschaftsrat 2013, 26-31 und Meyer-Guckel et al. 2015, 82f.

15 Hesser/Langfeldt 2017, 72f sowie Meyer-Guckel et al. 2015, 93 f.

16 Wissenschaftsrat 2013, S. $26 \mathrm{ff}$.
} 